\title{
Assessment of Available Transfer Capability in the Electricity Market
}

\author{
${ }^{* 1}$ Poonam Shende, ${ }^{2}$ B. Y. Bagde, ${ }^{3}$ B. S. Umre \\ ${ }^{1}$ Datta Meghe Institute of Engineering \& Technology and Research, Wardha, India \\ ${ }^{2}$ Yeshwantrao Chavan College of Engineering, Nagpur, India \\ ${ }^{3}$ Visvesvaraya National Institute of Technology, Nagpur, India \\ Email: Pshende93@gmail.com,by.bagde@gmail.com,bsumre@rediffmail.com
}

Received: 10th October 2019, Accepted: 31st January 2020, Published: 30th April 2020

\begin{abstract}
World over, the power system network has undergone restructuring process where vertically integrated utility is unbundled into generation, transmission and distribution sectors. This process has facilitated entry of private players in generation and distribution of power and introduced competitive environment in the power market. The available transfer capability (ATC) gives an assessment of probable transfer of power in the grid between different areas within the constraints of transmission system security and reliability. The estimation of ATC provides information on power transfer capability for current system condition and helps power marketers to make good decisions for power transactions between the utilities. In the proposed work, an attempt has been made to compare two methods of ATC calculation, i.e., repeated power flow (RPF) and power transfer distribution factor (PTDF) for bilateral transaction. Simulations carried out in MATLAB on IEEE 6 bus system shows that RPF is time consuming and slow. The PTDF method is then used to estimate ATC of a larger 39 bus New England test system for different system conditions of generation failure and line outage. For these conditions, critical line and critical amount of ATC is then determined.
\end{abstract}

\section{Keywords}

Available Transfer Capability (ATC), Power Transfer Distribution Factor (PTDF), Repeated Power Flow (RPF), DC Power Flow, AC Power Flow.

\section{Introduction}

With introduction of electricity act 2003 (IEA), Indian power sector has undergone restructuring process aimed at encouraging competitive environment in electricity market. The necessary licenses are provided to private players for generation and distribution of electricity while the government kept control over a transmission network. In 2004 electricity market take the first step towards restructuring by introducing open access (OA) and proceeded to form a short term day ahead market by Indian energy exchange (IEX) and Power Exchange India (PXIL) which is based on the ATC values. ATC gives an estimation of possible power transfer in an Indian grid between the different regions without violating the transmission system reliability and security. The decision of market splitting depends on congestion occurs in the transmission network hence ATC becomes the foundation of the electricity market [1].

North American Electric Reliability Council (NERC) provides a guideline to define and calculate the ATC in the power system and distinguish between the word 'capacity' and 'capability'. Normally capacity is term as device thermal limit where capability is referred to limitation depending on system topology. NERC report defines the principles of ATC calculation and allows individual systems to develop ATC determination procedure according to their principles. Some principles should be satisfied by the developed method. Such a developed method must find out the dependency of ATC at power injected point, the power extracted point and the power flow direction. Results obtained must be reasonably accurate and provide flow conditions according to time[2],[3].

According to NERC, ATC defines as "A measure of transfer capability in the physical transmission network for further commercial activity over and above already committed uses"

$A T C=-E T C+T T C-(T R M-C B M)$

Where, ETC is the 'Existing transmission commitment' which is an actual power transmitted from one area to another. TTC is 'Total transfer capability', the total amount of power safely exchange between two entities. TRM is the 'Transmission reliability margin'. CBM and TRM are reliability index which covers generation station uncertainties and transmission side uncertainties. Both reliability indexes are ignored to simplify the calculation method [4].

$A T C=-E T C+T T C$

In the open-access market, a transaction may be of two types bilateral transaction or multilateral transaction. A bilateral transaction consists of one buyer and one seller (even though there could be multiple points of utilization and injection). A group of multiple buyers and seller enters into a multilateral transaction [5],[6].

In the proposed work, Repeated power flow and DC power transfer distribution factor (DC-PTDF) method has been used to calculate ATC and these two methods is compared to conclude the faster method. DC-PTDF 
method considers DC power flow with some assumption. Variation in ATC for different system conditions such as generation outage and line outage is observed.

\section{Methodology}

\section{Repeated Power Flow}

RPF method [7] involved the continuous solution of the DC power flow equation. To calculate ATC for a bilateral contract, select a pair of source bus and sink bus pair in which one is a generator bus and another is a load bus. Base case power is calculated without any increase in generation power and load power which is used further to calculate the available transfer capability. Power generation at source bus is increased in step and the same amount of load power should be increased for each step at the sink bus.

The actual power flow through the transmission line is calculated in each step using DC power flow. This process is stop when any one transmission line reaches to its thermal limit. Consider $P^{\text {base }}$ as power injected at bus $a$ without any increase in generation or load and $P^{n e w}$ as power injected at bus $a$ obtain after any transmission line reaches its thermal line. Then,

$A T C=P^{\text {new }}-P^{\text {base }}$

The line reaches its thermal limit is the limiting factor for a power transaction in selected bilateral trading. The limiting factor is dependent upon the load level, generation dispatch, and network condition, etc.

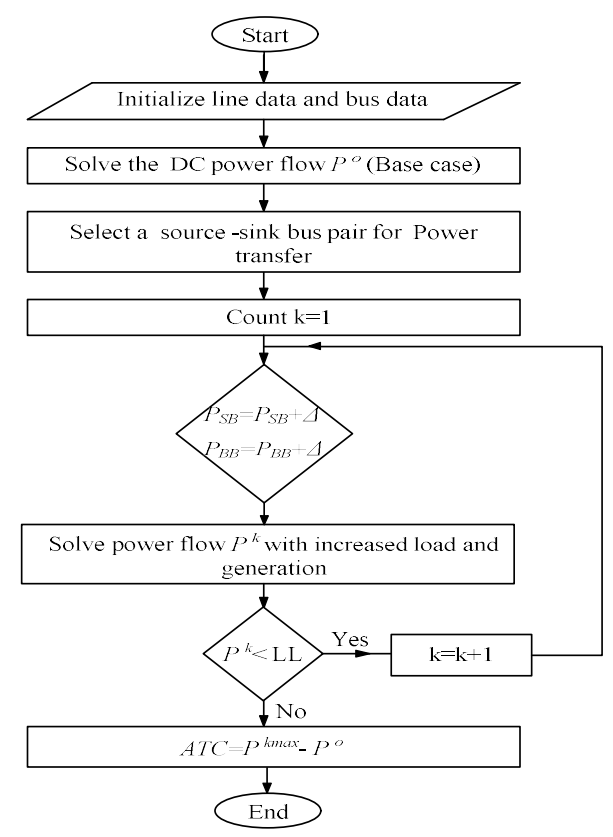

Fig. 1: Steps for RPF Algorithm

\section{A. Mathematical Formulation}

Consider,

$P_{a}^{0}$ be the base case power injected at bus $a$, when an algorithm of RPF is not started. Bus $a$ should be either a load bus or a generator bus.

$P_{a}^{k}$ be the power obtained at bus $a$ during the procedure of RPF after a certain steps $k$.

$k$ is the number of steps involved in RPF.

When any one of the transmission line of the system reaches to its thermal limit then,

$k=k^{\max }$

And RPF procedure stop at $k^{\max }$ iteration.

ATC for a selected bilateral transaction can be obtained as

$A T C=P_{a}^{k \max }-P_{a}^{0}$

Fig 1 describe the step by step procedure to determine the ATC using RPF method.

\section{Power Transfer Distribution Factor}

When a bilateral transaction is carried out between source- sink bus, power flow in different transmission line experience changes. These changes are defined by the PTDF values. From the power transfer distribution factor, 
the power operator can identify the power flow pattern of the transmission network [8].

\section{A. DC Power Flow}

DC power flow is obtained from AC power flow by considering three assumptions. The first assumption is to consider lossless transmission by ignoring the charging capacitance and resistance of the transmission line. The second assumption is considered voltage magnitude equal to $1 \mathrm{p} . \mathrm{u}$, and the third assumption is considered small variation in voltage angle and reactive power is eliminated. Hence real power is derived as a function of voltage angle.

\section{B. PTDF Calculation}

PTDF is the ratio of variation in the power flow in a transmission line $x$ which is in between bus $u$ and $v$ to power transmitted from $g$ bus to $f$ bus. DC power flow is used to derive the change in power flow in a line and hence approximate real power change is obtained.

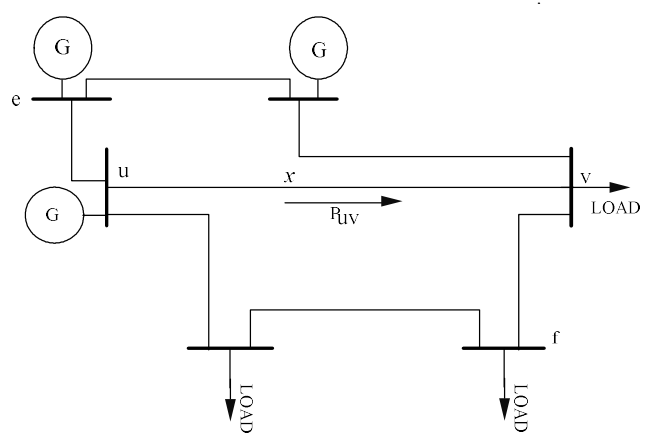

Fig. 2: Test System for PTDF Algorithm

$P T D F_{x, e f}=\frac{\Delta P_{x}}{\Delta P_{e f}}$

Where,

$\Delta P_{x}$ is variation in the actual power flow in line $x$

$\Delta P_{e f}$ is the magnitude of active power

$P T D F_{x, u v}$ is a sensitive factor of line $x$ for power transfer from bus $e$ to $f$

Mathematically PTDF is represented in terms of reactance as in (7)

$P T D F_{x, e f}=\frac{X_{u e}-X_{v e}-X_{u f}+X_{v f}}{x_{x}}$

Where,

$X_{u e}, X_{v e}, X_{u f}, X_{v f}$ are the values of bus reactance matrix

$x_{x}$ is the actual transmission line reactance

\section{ATC Calculation}

Estimation of ATC using PTDF algorithm is constructed based on DC power flow. ATC value for a particular transaction depends on the highest transfer limit of transmission line. Actual power transfer through a transmission line is estimated using (8)

$T L_{x}= \begin{cases}\frac{P_{x}^{\max }-P_{x}}{P T D F_{x}} & ; P T D F_{x}>0 \\ \infty & ; P T D F_{x}=0 \\ \frac{-P_{x}^{\max }-P_{x}}{P T D F_{x}} & ; P T D F_{x}<0\end{cases}$

Where,

$T L_{x}$ is the transfer limit of a line

$P_{x}^{\max }$ is the highest possible transmitting power that is the thermal constraint

$P_{i}$ is real power transmitted through the line.

In a transmission system, a line which has a less thermal limit with respect to other lines will be overloaded and selected as the ATC value for selected transaction. Mathematically, ATC value is determined as (9)

$A T C=\min \left(T L_{x}\right)$

Steps involved in PTDF algorithm to calculate ATC is described below: 
1. Identify the bus data and line data of the system

2. Calculate bus admittance matrix and eliminate reference bus number row and column to get bus reactance matrix.

3. Select a bilateral transaction and solve power flow

4. Determine PTDF matrix

5. Calculate the transfer limit as (8) for each line

6. Estimate ATC as (9)

\section{Results and Discussion}

\section{A. ATC Using RPF and PTDF Method}

To determine ATC using RPF algorithm and PTDF algorithm, IEEE 6 bus test system is used. System data of IEEE 6 bus test system is extracted from [4]. This system consists of three generator bus and three load bus hence six bilateral transactions is possible. Calculated ATC for each source-sink pair with its limiting factor and time required to complete the estimation of ATC is shown for PTDF based method and repeated power flow method in Table 1.

DC-PTDF based method requires less time as compare to RPF method as it does not require a repeated solution of the power flow equation and hence, further proposed work focus only on PTDF method.

\begin{tabular}{|c|c|c|c|c|c|}
\hline \multirow{2}{*}{$\begin{array}{c}\text { Source-Sink } \\
\text { Bus Pair }\end{array}$} & \multicolumn{4}{|c|}{ ATC in MW } & \multirow{2}{*}{ Limiting factor } \\
\cline { 2 - 5 } & RPF method & Time(sec) & $\begin{array}{c}\text { PTDF-based } \\
\text { method }\end{array}$ & Time(sec) & \\
\hline $2-4$ & 83.33 & $\mathbf{0 . 2 1}$ & 83.31 & $\mathbf{0 . 0 2 7 5}$ & $2-4$ \\
\hline $2-5$ & 65.85 & $\mathbf{0 . 1 4}$ & 65.83 & $\mathbf{0 . 0 2 7 7}$ & $4-5$ \\
\hline $2-6$ & 32.12 & $\mathbf{0 . 0 6}$ & 32.10 & $\mathbf{0 . 0 2 9 3}$ & $2-6$ \\
\hline $3-4$ & 89.10 & $\mathbf{0 . 1 6}$ & 89.08 & $\mathbf{0 . 0 2 4 6}$ & $2-3$ \\
\hline $3-5$ & 68.53 & $\mathbf{0 . 1 3}$ & 68.51 & $\mathbf{0 . 0 2 5 2}$ & $3-5$ \\
\hline $3-6$ & 63.26 & $\mathbf{0 . 1 1}$ & 63.24 & $\mathbf{0 . 0 2 5 6}$ & $3-6$ \\
\hline
\end{tabular}

Table 1: ATC Using RPF and PTDF Method

\section{B. ATC for a Different Condition}

In a deregulated power system, real-time operation and control are based on the power transaction. If the influence of different contingency on power transfer capability was known in advance then it would be beneficial for a power system operator to predict the possible power transaction and corrective action if congestion occurs in a future time.

39 bus New England system is considered to determine ATC for generator outage and load outage. Line data and bus data of the system is extracted from [9] ATC is calculated at normal operating condition using PTDF algorithm for a different bilateral transaction. The normal condition means the base case operation of the system without considering any contingency condition. Theoretically, there are 261 bilateral contracts possible for 39 bus New England system. The actual power flow through the transmission line is calculated by DC power flow using MATPOWER 6.0 software.

\section{ATC for Line Outage}

To examine the effect of transmission line failure on ATC for a particular source-sink pair some transmission line are randomly selected and disconnected. This transmission line are 1, 2, 8, 10, 21, 25, 28, 30, 40, 42. ATC was calculated for 30-3 source-sink bus pair. Base case ATC for 30-3 bus pair is 208.67 with line 3 (2-3) as a limiting factor. Table 2 and fig 3 shows the ATC for line outage. For different source-sink pair effect of line outage is different. A negative amount of ATC indicates the line is most congested.

\begin{tabular}{|c|c|c|c|}
\hline Line No. & Line out & ATC(in MW) & Limiting factor \\
\hline Base case & -- & 208.67 & $2-3$ \\
\hline 1 & $1-2$ & 29.72 & $2-3$ \\
\hline 2 & $1-39$ & 127.65 & $2-3$ \\
\hline 8 & $4-5$ & 170.78 & $2-3$ \\
\hline 10 & $5-6$ & 196.45 & $2-3$ \\
\hline 21 & $12-11$ & 208.65 & $2-3$ \\
\hline 25 & $15-16$ & 186.34 & $2-3$ \\
\hline 28 & $16-21$ & 208.67 & $2-3$ \\
\hline 30 & $17-18$ & 88.68 & $2-3$ \\
\hline 40 & $25-26$ & 148.91 & $2-3$ \\
\hline 42 & $26-27$ & -73.18 & $2-3$ \\
\hline
\end{tabular}

Table 2: ATC for Line Outage 


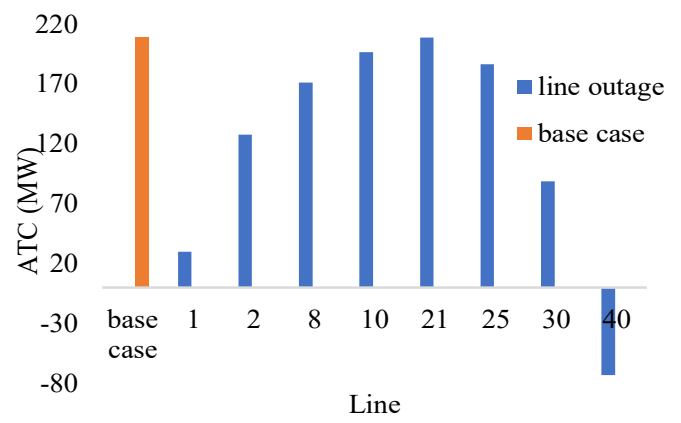

\section{ATC for Generator Failure}

Fig. 3: ATC for Line Outage

To examine the effect of generator outage on ATC, some generator was disconnected and ATC is calculated for a particular source-sink bus pair. Some generators $1,3,5,7$, and 9 are randomly selected and disconnected. ATC was calculated for 30-3 source-sink bus pair. Base case ATC for 30-3 bus pair is 208.67 with line 3 (2-3) as a limiting factor. Table 3 and fig 4 shows the ATC for generator outage.

Power flow through each transmission line (power flow pattern) will change with each disconnected generator because, slack bus (generator bus) takes a responsibility to supply power when generation is out of service and hence ATC will change.

\begin{tabular}{|c|c|c|}
\hline Generator out & ATC(MW) & Limiting factor \\
\hline Gen 1 & 406.45 & $2-3$ \\
\hline Gen 3 & 198.46 & $2-3$ \\
\hline Gen 5 & 206.19 & $2-3$ \\
\hline Gen 7 & 205.94 & $2-3$ \\
\hline Gen 9 & -88.33 & $4-5$ \\
\hline
\end{tabular}

Table 3: ATC for Generation Outage

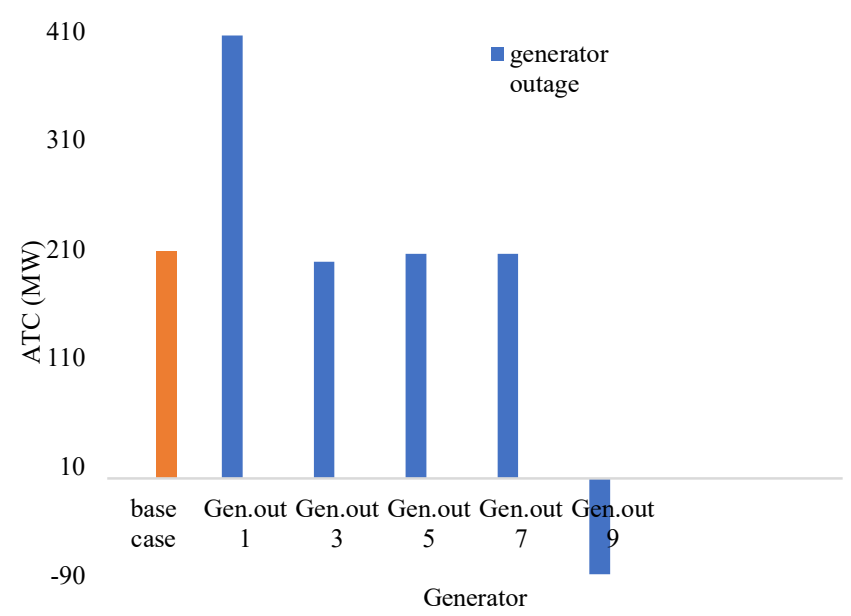

Fig. 4: ATC for Generator Outage

\section{Conclusion}

ATC is calculated using RPF method and PTDF-based method. RPF method requires repeated solutions of power flow equations. Hence, it becomes time consuming for a large system. On the other hand PTDF-based method does not require repeated solution method Hence, it is faster than the RPF method. For 39 bus New England test system, ATC is evaluated for transmission line outage and generation outage. With a change in generator failure and line outage critical line is determined with a critical amount of ATC which helps system operators for better planning and secure operation of the system.

The results obtained for ATC can be extended for demand side management. ATC can be used to determine the 
optimal buses and hours to implement the demand response program which helps system operator to apply different tariffs for peak demand. This reduces the consumption thereby relieving congestion of lines.

\section{References}

1. P. Pande, S. Kumar, A. Sinha, "Total transfer capability calculation using modified repeated power flow," 2015 Annual IEEE Indian Conference (INDICON), New Delhi, India, 17-20 Dec 2015.

2. M. Bhaskar, J, Jimoh, "Available transfer capability calculation using PTDF and implementation of optimal power flow in power marketing," IEEE International Conference on renewable energy research and applications(ICREA), Birmingham, UK, 20-23 Nov 2016

3. G. Hamoud, “Assessment of available transfer capability of transmission system, ” IEEE Transactions on power system, vol. 15, no. 1, Feb 2000.

4. P. A. Shende, B. Y. Bagde, B. S. Umre, "Estimation of available transfer capability for bilateral trading transactions," Scopus: International journal of innovation technology and exploring engineering (IJITEE),vol. 8 issue-8S3, pp 307-311 June 2019

5. P. Venkatesh, R. Ganadass, N. Padhy, "Available transfer capability determination using power transfer distribution factors," International journal of emerging electric power system, vol.1, issue 2, pp 1-14 Jan 2004.

6. A. Kumar and M. Kumar, "Available transfer capability determination using power transfer distribution factors," International Journal of Information and. Computation Technology, vol. 3, no. 11, pp. 1035-1040, 2013.

7. S. Deke, "Available transfer capability calculations considering outages," IEEE International Conference on power and embedded drive control, Chennai, India, 16-18 march 2017.

8. E. Dehnavi, H. Abdi, "Determine optimal buses for implementing demand response as an effective congestion management method," IEEE Transaction on power system, vol.32 no.2, March 2017

9. M. karthiga, S. Raja, P. Venkatesh, "Enhancement of available transfer capability using TCSC devices in deregulated power market," IEEE conference on innovation in power and advance computing techniques (i-PACT), 21-22 April 2017.

10. M. Patil, A. Girgis, "New iterative method for available transfer capability calculation," IEEE Conference on power and energy society general meeting, Detroit, USA, 24-29 July 2011.

11. A. Pillay, S. Prabhakar Karthikeyan, and D. P. Kothari, "Congestion management in power systemsA review," International Journal Of Electrical Power and Energy Systems, vol. 70, pp. 83-90, Sep. 2015.

12. R. D. Zimmerman, C. E. Murillo-Sanchez, and D. Gan, "MATPOWER: A MATLAB power system simulation package." (Mar. 2015). [Online]. Available: http://www.pserc.cornell.edu/matpower/

13. R. Zimmerman, C. Murillo-Sanchez, R. Thomas, "Matpower: Steady-state operations, planning and analysis tools for power systems research and education," IEEE Transactions on power systems, vol. 26, no. 1, pp. 12-19, Feb. 2011.

14. M. Patil, "Review of available transmission capability (ATC) calculation methods," IEEE Conference on power system, Clemson, SC, USA, 10-13 march. 